\title{
基于辐射传输模型的高分二号影像大气校正方法研究
}

\author{
曹红业 ${ }^{1}$, 张天棋 ${ }^{2}$ \\ (1. 长安大学 地质工程与测绘学院, 陕西 西安 710061; 2. 西南科技大学 环境与资源学院, 四川 绵阳 621010)
}

摘要: 高分二号卫星的成功发射, 标志着我国遥感卫星进入了亚米级高空间分辨率时代, 遥感影像在 定量反演, 地物识别和变化分析等领域将有重要作用。大气校正的精度是影响其定量化应用的重要因 素。由于高分二号遥感数据缺乏短波红外波段，无法采用暗像元法进行大气校正。提出一种基于辐射 传输模型的高分二号影像大气校正方法, 利用 6S (second simulation of the satellite signal in the solar spectrum）辐射传输模型建立大气校正系数查找表，利用同步 MODIS 影像数据结合改进后的暗像元 方法反演气溶胶光学厚度, 确定大气校正系数, 消除高分二号影像大气分子和气溶胶等的吸收和散射 的影响, 实现 GF-2 数据的大气校正。选取地表平坦均一的敦煌辐射校正场作为实验区, 通过同步的 实测数据对校正结果进行精度评价, 并且比较大气校正前后归一化植被指数 NDVI。结果表明: 最小 相对误差仅为 $0.9 \%$, 大气校正之后影像数据更真实地反映了地物的反射特性; 大气校正后的 NDVI 大大增强了植被信息反差, 突出了 GF-2 卫星传感器的植被信息区分能力。

关键词: 辐射传输模型; GF-2 影像; 大气校正; 查找表; 气溶胶光学厚度; $6 \mathrm{~S}$

中图分类号：TP751 文献标识码：A 文章编号：1001-8891(2020)06-0534-08

\section{Atmospheric Correction Algorithm for GF-2 Image Based On a Radiative Transfer Model}

\author{
CAO Hongye $^{1}$, ZHANG Tianqi ${ }^{2}$ \\ (1. School of Geological Engineering and Surveying Engineering, Chang'an University, Xi'an 710061, China; \\ 2. School of Environment and Resource, Southwest University of Science and Technology, Mianyang 621010, China)
}

\begin{abstract}
The successful launch of the GF-2 satellite indicates that China's remote sensing satellites have entered the era of high spatial resolution of the sub-meter level. Remote sensing images will play an important role in quantitative inversion, ground object recognition, and change analysis. The accuracy of its atmospheric correction is an important factor that affects its quantitative application. Due to the lack of a short-wave infrared band in GF-2, it is impossible to use a dark pixel method for atmospheric correction. A method of atmospheric correction for the GF-2 image based on a radiation transfer model is proposed. The atmospheric correction coefficient lookup table is established by using 6S (Second Simulation of the Satellite Signal in the Solar Spectrum) radiation transfer model. The aerosol optical thickness (AOT) is retrieved by combining synchronous MODIS image data with an improved dark pixel method. The atmospheric correction parameters are determined to eliminate the influence of absorption and scattering of atmospheric molecules and aerosols in the GF-2 image and to achieve atmospheric correction of GF-2 data. Dunhuang radiation correction field with a flat and uniform surface is selected as the experimental area. The accuracy of the correction results is evaluated by synchronous measured data, and the normalized difference vegetation index (NDVI) before and after atmospheric correction is compared. The results show that the minimum relative error is only $0.9 \%$. The image data after atmospheric correction can accurately reflect the reflection characteristics of ground objects. NDVI after atmospheric correction greatly enhances the contrast of vegetation information and highlights the ability of vegetation information discrimination of the GF-2 satellite sensor.
\end{abstract}

Key words: radiation transfer model, GF-2 image, atmospheric correction, look-up table, aerosol optical depth(AOD), $6 \mathrm{~S}$ 


\section{0 引言}

2014 年 8 月 19 日 “高分二号” (GF-2) 卫星发射 成功, 标志着我国遥感卫星进入亚米级高空间分辨时 代 ${ }^{[1]}$ 。该卫星的成功发射, 为我国土地利用动态监测、 矿产资源调查、城乡规划监测评价、交通路网规划、 森林资源调查、荒漠化监测等诸多方面应用提供了新 的数据源。如表 1 所示, 融合后的 GF-2 卫星空间分 辨率优于 $1 \mathrm{~m}$, 共有蓝、绿、红和近红外 4 个多光谱 波段, 成像幅宽为 $45 \mathrm{~km}$, 具有 $\pm 35^{\circ}$ 的侧摆角, 可以 实现 $69 \mathrm{~d}$ 内对全球的观测覆盖, 以及 $5 \mathrm{~d}$ 内对地球表 面任意区域的重复观测。

电磁波在传播过程中受到大气分子和气溶胶等大 气成分的吸收和散射的影响, 使传感器获得的电磁信 号中带有一定的非目标地物信息, 传感器接收到的地 物信息不能真实地反应地表, 严重影响其定量化应用, 这就需要对该过程进行大气校正, 消除非目标地物信 息, 获得真实的地表反射率信息 ${ }^{[2-3]}$ 。因此大气校正是 GF-2 影像数据地表参数定量反演的一个必备环节。

目前, 大气校正的方法主要有基于图像特征模 型、地表实测线性回归模型法和辐射传输模型法 3 种 ${ }^{[4-6]}$ 。其中辐射传输法是利用复杂的辐射传输原理 建立起来的一种方法, 利用辐射传输模型反演地物反 射率可以较合理地描述大气散射、吸收等过程。自 1972 年 Turner 与 Spencer 通过模拟大气-地表系统来 评估大气影响 ${ }^{[7]}$, 成为最早的大气辐射传输模型之一, 在诸多大气校正方法中具有高精度和高普适性。

GF-2 卫星数据业务化应用要求基于遥感影像来 反演大气参数, 进而完成整个大气校正过程。一些传 感器专门设置了用于反演大气参数的波段, 如 MODIS 设置了 15 和 16 波段用于反演气溶胶 ${ }^{[8]}, 17,18$ 和 19

表 1 高分二号卫星有效荷载技术指标

Table 1 Satellite payload technology index of GF-2

\begin{tabular}{|c|c|c|c|c|c|c|}
\hline Satellite payload & $\begin{array}{c}\text { Band } \\
\text { number }\end{array}$ & $\begin{array}{c}\text { Band } \\
\text { range/ } \mu \mathrm{m}\end{array}$ & $\begin{array}{c}\text { Spatial } \\
\text { resolution/m }\end{array}$ & Width $/ \mathrm{km}$ & $\begin{array}{c}\text { Side swing } \\
\text { angle }\end{array}$ & $\begin{array}{c}\text { Revisited } \\
\text { days }\end{array}$ \\
\hline \multirow{5}{*}{$\begin{array}{c}\text { Panchromatic and } \\
\text { multispectral } \\
\text { sensors(PMS) }\end{array}$} & 1 & $0.45-0.90$ & 1 & \multirow{5}{*}{45} & \multirow{5}{*}{ $\pm 35^{\circ}$} & \multirow{5}{*}{5} \\
\hline & 2 & $0.45-0.52$ & \multirow{4}{*}{4} & & & \\
\hline & 3 & $0.52-0.59$ & & & & \\
\hline & 4 & $0.63-0.69$ & & & & \\
\hline & 5 & $0.77-0.89$ & & & & \\
\hline
\end{tabular}

将(1)式经过转换, 可以得到均匀朗伯表面地表反射率 $\rho\left(\tau_{\mathrm{a}}, \mu_{\mathrm{s}}, \mu_{\mathrm{v}}, \phi\right)$ :

$$
\rho\left(\tau_{\mathrm{a}}, \mu_{\mathrm{s}}, \mu_{\mathrm{v}}, \phi\right)=\frac{\rho_{\mathrm{t}}\left(\tau_{\mathrm{a}}, \mu_{\mathrm{s}}, \mu_{\mathrm{v}}, \phi\right)-\rho_{\mathrm{a}}\left(\tau_{\mathrm{a}}, \mu_{\mathrm{s}}, \mu_{\mathrm{v}}, \phi\right)}{T^{\downarrow}\left(\mu_{\mathrm{s}}\right) T^{\uparrow}\left(\mu_{\mathrm{v}}\right)+\left(\rho_{\mathrm{t}}\left(\tau_{\mathrm{a}}, \mu_{\mathrm{s}}, \mu_{\mathrm{v}}, \phi\right)-\rho_{\mathrm{a}}\left(\tau_{\mathrm{a}}, \mu_{\mathrm{s}}, \mu_{\mathrm{v}}, \phi\right)\right) S}
$$

式中: $\rho_{\mathrm{t}}\left(\tau_{\mathrm{a}}, \mu_{\mathrm{s}}, \mu_{\mathrm{v}}, \phi\right)$ 为 GF-2 表观反射率; $\rho_{\mathrm{a}}\left(\tau_{\mathrm{a}}, \mu_{\mathrm{s}}, \mu_{\mathrm{v}}, \phi\right)$ 为瑞利散射和气溶胶散射引起的大气路径辐射表观反 射率; $T^{\downarrow}\left(\mu_{\mathrm{s}}\right) T^{\uparrow}\left(\mu_{\mathrm{v}}\right)$ 为大气吸收构成的上、下行辐射透过率乘积。
波段用于反演大气水汽含量等 ${ }^{[9]}$ 。但高分二号影像没 有设置用于反演大气参数的波段, 给高分二号影像的

本文提出一种基于同步 MODIS 数据辅助的高分 二号多光谱数据的大气校正算法。使用 MODIS 同步 数据反演气溶胶光学厚度, 并建立高分二号影像大气 校正系数查找表, 对高分二号影像进行大气校正。在 对一景高分二号影像进行大气校正后, 从典型地物光 谱数据、归一化植被指数 (normalized difference vegetation index, NDVI) 两方面, 探讨了 GF-2 影像大

由于 GF-2 卫星 CCD 相机属于典型的 4 波段传感 器, 相机所设置的 4 个波段均处于大气窗口, 水汽、 臭氧等气体的影响较小, 可忽略不计。假设陆地表面 是均匀朗伯表面, 大气垂直均匀变化的条件下, 传感

$$
\begin{aligned}
& L\left(\tau_{\mathrm{a}}, \mu_{\mathrm{s}}, \mu_{\mathrm{v}}, \phi\right)=L_{\mathrm{a}}\left(\tau_{\mathrm{a}}, \mu_{\mathrm{s}}, \mu_{\mathrm{v}}, \phi\right)+ \\
& \quad F_{\mathrm{d}}\left(\tau_{\mathrm{a}}, \mu_{\mathrm{s}}\right) T^{\downarrow}\left(\mu_{\mathrm{s}}\right) T^{\uparrow}\left(\mu_{\mathrm{v}}\right) \rho /\left[1-S\left(\tau_{\mathrm{a}}\right) \rho\right]
\end{aligned}
$$

式中: 传感器接收到的表观辐亮度 $L\left(\tau_{\mathrm{a}}, \mu_{\mathrm{s}}, \mu_{\mathrm{v}}, \phi\right)$ 由大 气路径辐射 $L_{\mathrm{a}}\left(\tau_{\mathrm{a}}, \mu_{\mathrm{s}}, \mu_{\mathrm{v}}, \phi\right)$ 和地表反射辐射部分组成; $F_{\mathrm{d}}\left(\tau_{\mathrm{a}}, \mu_{\mathrm{s}}\right)$ 为大气顶部与太阳光垂直方向的入射辐射 通量密度; $S$ 为大气半球反射率; $\rho$ 表示地表反射率; $T^{\uparrow}\left(\mu_{\mathrm{v}}\right)=\mathrm{e}^{-\tau / \mu_{\mathrm{v}}}+t_{\mathrm{d}}^{\uparrow}\left(\theta_{\mathrm{v}}\right) ; T^{\downarrow}\left(\mu_{\mathrm{s}}\right)=\mathrm{e}^{-\tau / \mu_{\mathrm{s}}}+t_{\mathrm{d}}^{\downarrow}\left(\theta_{\mathrm{s}}\right)$ 分别为大 气吸收构成的上、下行辐射透过率; $t_{\mathrm{d}}^{\uparrow}\left(\theta_{\mathrm{v}}\right), t_{\mathrm{d}}^{\downarrow}\left(\theta_{\mathrm{s}}\right)$ 表 示上、下行散射辐射透过率因子; $\mathrm{e}^{-\tau / \mu_{\mathrm{v}}} 、 \mathrm{e}^{-\tau / \mu_{\mathrm{s}}}$ 分别 为大气对太阳下行、上行直接辐射的透射因子; $\mu_{\mathrm{s}}$ 和 $\mu_{\mathrm{v}}$ 分别表示太阳天顶角和传感器天顶角的余弦值, $\phi$ 表示太阳方位角和传感器方位角的相对方位角 ${ }^{[10]}$ 。 大气校正带来了很大的困难。 气校正的效果。

\section{1 大气校正原理}


大气校正过程可以分为如下 3 步:

(1)利用暗像元法获取气溶胶光学厚度 ${ }^{[11]}$;

(2)建立大气校正查找表, 计算辐射传输方程中相 应的大气参数和观测条件下的大气半球反射率 $S$, 大 气路径辐射表观反射率 $\rho_{\mathrm{a}}\left(\tau_{\mathrm{a}}, \mu_{\mathrm{s}}, \mu_{\mathrm{v}}, \phi\right)$ 和上、下行辐射

透过率乘积 $T^{\uparrow}\left(\mu_{\mathrm{v}}\right) T^{\downarrow}\left(\mu_{\mathrm{s}}\right) 3$ 个大气校正系数;

(3)将(2)中得到的 3 个大气校正系数带入公式(2) 中, 利用 GF-2 影像数据获得的表观反射率 $\rho_{\mathrm{t}}\left(\tau_{\mathrm{a}}, \mu_{\mathrm{s}}, \mu_{\mathrm{v}}, \phi\right)$ 可以求得地表反射率 $\rho\left(\tau_{\mathrm{a}}, \mu_{\mathrm{s}}, \mu_{\mathrm{v}}, \phi\right)$ 。

\section{1 数据预处理}

\subsubsection{GF-2 辐射定标}

根据传感器各波段辐射定标系数将 DN 值转换为 表观辐亮度, 即传感器入瞳处的辐射亮度值, 辐射定 标公式为:

$$
L=\text { Gain } \cdot \mathrm{DN}+\text { Offset }
$$

式中: $L$ 为传感器辐射亮度值; Gain 和 Offset 分别为 影像绝对定标系数增益和偏移, 数据源自中国资源卫 星应用中心网站 (www.cresda.com)。高分二号卫星影 像各波段 2015 年绝对辐射定标系数如表 2 所示 (GF-2 的 Offset 值全部为零)。

\section{表 22015 年 GF-2 PMS 辐射定标系数}

Table 2 Radiation calibration coefficient of GF-2 PMS in 2015

\begin{tabular}{ccccc}
\hline $\begin{array}{c}\text { Satellite } \\
\text { payload }\end{array}$ & Band 1 & Band 2 & Band 3 & Band 4 \\
\hline PMS1 & 0.1457 & 0.1604 & 0.1550 & 0.1731 \\
PMS2 & 0.1761 & 0.1843 & 0.1677 & 0.1830 \\
\hline
\end{tabular}

1.1.2 GF-2 影像表观反射率计算

在上述表观辐射亮度计算基础上, 根据如下计算 公式得到大气层顶表观反射率:

$$
\rho_{\mathrm{t}}=\frac{\pi \cdot L \cdot d^{2}}{\mathrm{ESUN} \cdot \cos \theta_{\mathrm{s}}}
$$

式中: $d$ 为日地距离校正因子, 如表 3 所示, 计算时 可对表中的数值进行插值获得; $L$ 为表观辐亮度; $\theta_{\mathrm{s}}$ 为太阳天顶角。
ESUN 是大气上界太阳光辐照度, 通过 GF-2 影 像光谱响应函数 (如图 1 所示) 和太阳光谱辐照度数 据根据式(5)积分计算, GF-2 影像光谱响应函数可在 中国资源卫星应用中心网站获取, 太阳光谱辐照度数 据采用张璐等人 ${ }^{[12]}$ 推荐的 Wehrli 数据, 如图 2 所示。 ESUN 计算公式如下:

$$
\operatorname{ESUN}=\frac{\int_{\lambda_{1}}^{\lambda_{2}} E(\lambda) \cdot f(\lambda) \mathrm{d} \lambda}{\int_{\lambda_{1}}^{\lambda_{2}} f(\lambda) \mathrm{d} \lambda}
$$

式中: $E(\lambda)$ 为波长 $\lambda$ 处的大气层外太阳光谱辐照度; $f(\lambda)$ 为波长 $\lambda$ 处的光谱响应函数; $\lambda_{1}$ 和 $\lambda_{2}$ 为积分波段范围 的上下限波长。

\section{2 计算气溶胶光学厚度}

由于 MODIS 短波红外波段（2.12 $\mu \mathrm{m})$ 具有较高 的大气透过率且对气溶胶的光学特性敏感。本文利用 暗像元法求取气溶胶光学厚度值, 暗像元法是由 Kaufman 等人 ${ }^{[13]}$ 提出, 原理是 MODIS $2.1 \mu \mathrm{m}$ 通道的 数据受气溶胶的影响比较小, 可以较好地反映地面的 特征, 因而其表观反射率与其地面反射率近似相等。 影像上植被覆盖区、水体等暗像元的红 $(0.660 \mu \mathrm{m})$ 、 蓝波段 $(0.470 \mu \mathrm{m})$ 的地表反射率可以通过 $2.1 \mu \mathrm{m}$ 波 段的地表反射率它们之间的线性关系估算出，

$\rho_{0.47}^{\mathrm{s}}=\rho_{2.1}^{*} / 4, \rho_{0.66}^{\mathrm{s}}=\rho_{2.1}^{*} / 2$ 。但是该方法没有考虑植 被指数和散射角对地表反射率的影像, Robert 等 ${ }^{[14]}$ 提出了改进的暗像元法, 其具体算法如下:

$$
\left\{\begin{array}{l}
\rho_{0.66}^{\mathrm{s}}=\rho_{2.1}^{\mathrm{m}} \cdot \text { slope }_{0.66 / 2.1}+y \text { int }_{0.66 / 2.1} \\
\rho_{0.47}^{\mathrm{s}}=\rho_{0.66}^{\mathrm{s}} \cdot \text { slope }_{0.47 / 2.1}+y \text { int }_{0.47 / 2.1}
\end{array}\right.
$$

$$
\text { 式中: }\left\{\begin{array}{l}
\text { slope }_{0.66 / 2.1}=\operatorname{slope}_{0.66 / 2.1} \mathrm{NDVI}_{\mathrm{ISwR}}+0.002 \Theta-0.27 \\
\Theta=\cos ^{-1}\left(-\cos \theta_{\mathrm{S}} \cos \theta_{\mathrm{V}}+\sin \theta_{\mathrm{S}} \sin \theta_{\mathrm{V}} \cos \phi\right) \\
y \operatorname{int}_{0.66 / 2.1}=0.00025 \Theta+0.033 \\
\operatorname{slope}_{0.47 / 2.1}=0.49 \\
y \operatorname{int}_{0.47 / 2.1}=0.005
\end{array}\right.
$$

\begin{tabular}{|c|c|c|c|c|c|c|c|c|c|}
\hline Days & Distance & Days & Distance & Days & Distance & Days & Distance & Days & Distance \\
\hline 1 & 0.98331 & 74 & 0.99446 & 152 & 1.01403 & 227 & 1.01281 & 305 & 0.99253 \\
\hline 15 & 0.98365 & 91 & 0.99926 & 166 & 1.01577 & 242 & 1.00969 & 319 & 0.98916 \\
\hline 32 & 0.98536 & 106 & 1.00353 & 182 & 1.01667 & 258 & 1.00566 & 335 & 0.98608 \\
\hline 46 & 0.98774 & 121 & 1.00756 & 196 & 1.01646 & 274 & 1.00119 & 349 & 0.98426 \\
\hline 60 & 0.99084 & 135 & 1.01087 & 213 & 1.01497 & 288 & 0.99718 & 365 & 0.98333 \\
\hline
\end{tabular}

表 3 日地距离 Table 3 Solar-earth distance

天文单位(astronomical units) 


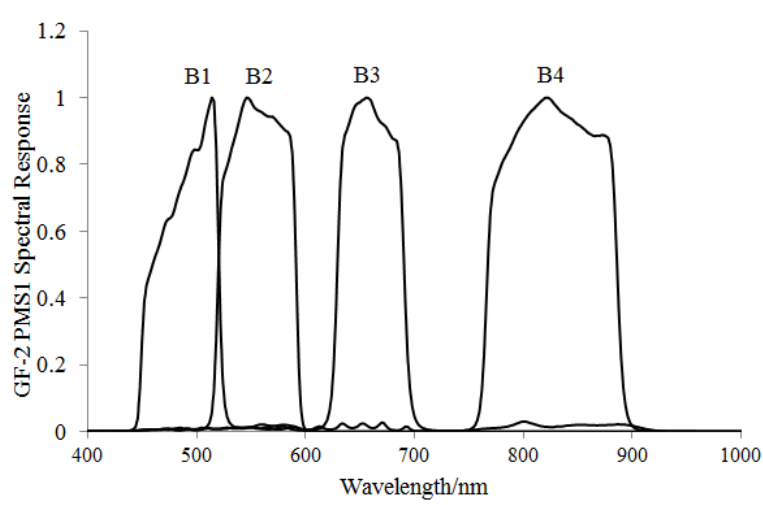

图 $1 \mathrm{GF}-2$ 光谱响应函数

Fig.1 Spectral response function of GF-2

$$
\text { slope }_{0.66 / 2.1}^{\mathrm{NDI}_{\text {SWIR }}}=\left\{\begin{array}{l}
0.48, \mathrm{NDVI}_{\text {SWIR }}<0.25 \\
0.58, \mathrm{NDVI}_{\text {SWIR }}>0.25 \\
0.48+0.2 \cdot\left(\mathrm{NDVI}_{\text {SWIR }}-0.25\right), \\
0.25 \leq \mathrm{NDVI}_{\mathrm{SWIR}} \leq 0.75
\end{array}\right.
$$

$$
\mathrm{NDVI}_{\text {SWIR }}=\left(\rho_{1.2}^{\mathrm{m}}-\rho_{2.1}^{\mathrm{m}}\right) /\left(\rho_{1.2}^{\mathrm{m}}+\rho_{2.1}^{\mathrm{m}}\right)
$$

式中: $\rho_{1.2}^{\mathrm{m}} 、 \rho_{2.1}^{\mathrm{m}}$ 分别表示 $1.2 \mu \mathrm{m}$ 和 $2.1 \mu \mathrm{m}$ 通道处的 表观反射率; $\rho_{0.47}^{\mathrm{s}} 、 \rho_{0.66}^{\mathrm{s}}$ 分别为 $0.47 \mu \mathrm{m}$ 和 $0.66 \mu \mathrm{m}$ 波 段的地表反射率; $\Theta$ 表示散射角; $\theta_{\mathrm{S}} 、 \theta_{\mathrm{V}} 、 \phi$ 表示太阳 天顶角、卫星天顶角和相对方位角。

利用 $2.1 \mu \mathrm{m}$ 通道和 $1.2 \mu \mathrm{m}$ 通道的表观反射率可 以准确地估算出红蓝通道的地表反射率, 利用 $6 \mathrm{~S}$ 模 型计算不同大气气溶胶模式和观测条件下, 气溶胶光 学厚度 $\tau_{\mathrm{a}}(\mathrm{red})$ 和 $\tau_{\mathrm{a}}$ (blue) 和大气半球反射率 $S$, 大气程 辐射 $\rho_{\mathrm{a}}$ 和大气吸收构成的辐射透过率 $T$ 等大气校正系 数之间的关系, 据此建立气溶胶光学厚度查找表, 其 中与实际表观反射率相等或最相近的模拟表观反射 率对应下的气溶胶光学厚度即为最终结果, 可以求得 红蓝波段气溶胶光学厚度 $\tau_{\mathrm{a}}(\mathrm{red})$ 和 $\tau_{\mathrm{a}}($ blue $)$ 。

结合气溶胶光学厚度随波长变化的指数关系 Angstrom 公式 ${ }^{[15]}, \tau_{\mathrm{a}}(\lambda)=\beta \cdot \lambda_{i}^{-\alpha}$ ，其中 $\alpha$ 表示 Angstrom 波长指数, 反映了气溶胶大小粒子的比例, $\alpha$ 越大说明气溶胶粒子尺度越大; $\beta$ 表示大气浑浊度参 数, 反映了大气气溶胶粒子的浓度。将红蓝波段的气 溶胶光学厚度 $\tau_{\mathrm{a}}(\mathrm{red})$ 和 $\tau_{\mathrm{a}}$ (blue) 代入 Angstrom 公式中 确定参数 $\alpha$ 和 $\beta$, 得到其他波段的气溶胶光学厚度。

\section{3 构建大气校正系数查找表}

利用 $6 \mathrm{~S}$ 辐射传输模型, 在输入参数中设置多个 不同的卫星观测几何、太阳观测几何、卫星天顶角、 大气模式、气溶胶光学厚度、地面高度等变量。其中, 卫星观测几何、太阳观测几何、地面高度等参数从

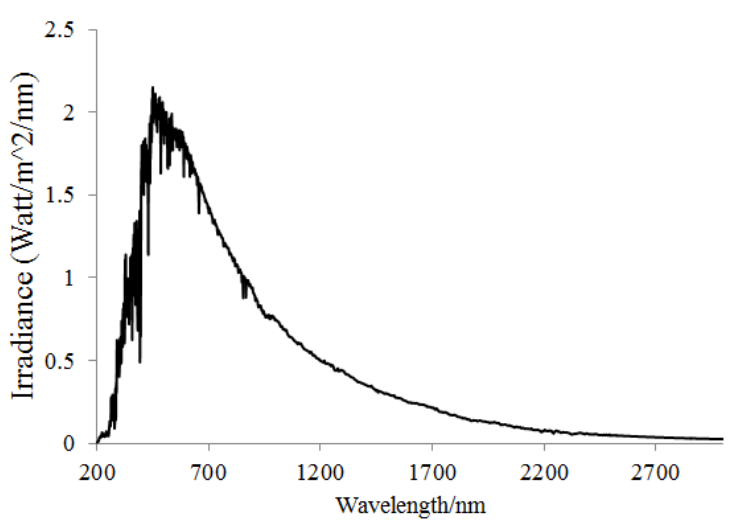

图 2 Wehrli 太阳光谱辐照度

Fig.2 Solar spectral irradiance of Wehrli

GF-2 影像对应的元数据文件中获取; 大气模式包括热 带（Tropical, T)、中纬度夏季（Mid-latitude Summer, WLS）、中纬度冬季（Mid-latitude Winter, MLW）、近 极地冬季 ( Subarctic Winter, SAW), 近极地夏季 (Subarctic Summer, SAS) 等几种大气模式, 根据 GF-2 影像的成像时间及纬度决定。由于 $6 \mathrm{~S}$ 模型本身并未 附带 GF-2 传感器的光谱响应函数, 因此, 需要将其 光谱响应函数重采样为 $2.5 \mathrm{~nm}$ 分辨率, 输入到 $6 \mathrm{~S}$ 模 型中。

由于研究区域地物反射率均低于 0.6 , 为了便于 建立查找表, 本文假设 3 个地表反射率 $\rho$ 为 $0,0.2$, 0.5 , 选定了大气模式后, 从 GF-2 影像元文件中读取 太阳天顶角、太阳方位角、卫星天顶角、卫星方位角 4 个角度数据, 输入 1.2 节求解的气溶胶光学厚度, 连续运行 $6 \mathrm{~S}$ 程序 3 次, 得到其 3 个模拟表观反射率 分别为 $\rho_{0.0}{ }^{\mathrm{t}}, \rho_{0.2}{ }^{\mathrm{t}}, \rho_{0.5}{ }^{\mathrm{t}}$, 将结果代入公式(6)中, 得到 一个关于大气程辐射项 $\rho_{\mathrm{a}}$, 大气吸收构成的辐射透过 率 $T$, 大气半球反射率 $S$ 的三元一次方程组, 求解该 方程组, 得到如下用模拟表观反射率为参数表示的 解:

$$
\left\{\begin{array}{l}
\rho_{a}=\rho_{0.0}^{t} \\
S=\frac{2 \rho_{0.5}^{t}-5 \rho_{0.2}^{t}+3 \rho_{0.0}^{t}}{\rho_{0.5}^{t}-\rho_{0.2}^{t}} \\
T=\left(\rho_{0.2}^{t}-\rho_{0.0}^{t}\right)(5-S)
\end{array}\right.
$$

通过设置上述参数, 建立一个关于辐射传输方程 中的大气程辐射项 $\rho_{\mathrm{a}}$ 、大气吸收构成的辐射透过率 $T$ 、 大气半球反射率 $S$ 、太阳天顶角 $\theta_{\mathrm{s}}$ 、卫星天顶角 $\theta_{\mathrm{v}}$ 、 太阳方位角和卫星方位角的 7 维查找表, 其他值在它 们之间进行线性插值。

\section{4 基于查找表的 GF-2 大气校正}

基于 MODIS 数据反演得到的气溶胶光学厚度, 
结合大气校正查找表逐像元插值, 计算出对应的大气 校正系数, 进而计算出地表反射率。大气校正流程如 图 3 所示。

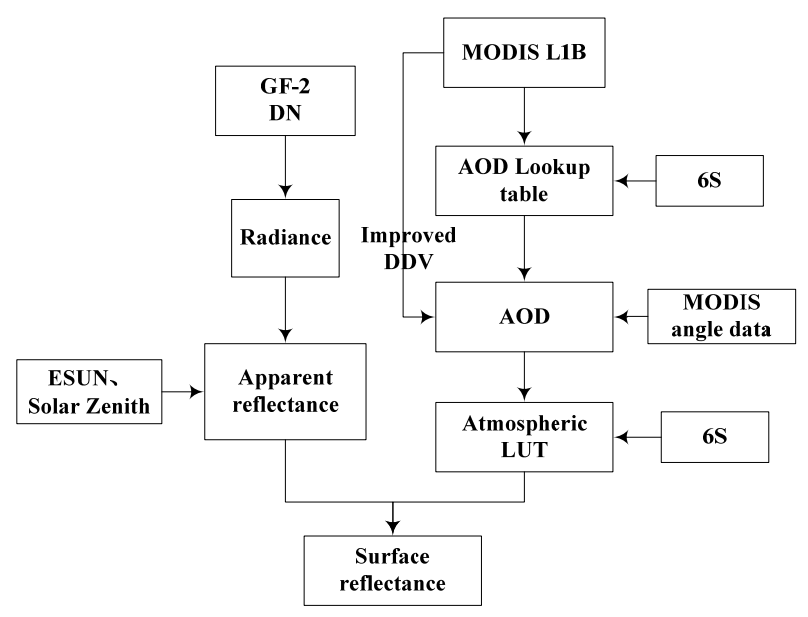

图 3 高分二号影像大气校正流程图

Fig.3 Flowchart of atmospheric correction of GF-2 image

对 MODIS 进行去 “蝴蝶” 效应, 几何校正, 辐 射定标等预处理工作 ${ }^{[16-18]}$, 使用 IDL 语言编程调用 $6 \mathrm{~S}$ Fortran 程序, 建立气溶胶光学厚度查找表, 使用 改进后的暗像元法逐像元反演气溶胶光学厚度 ${ }^{[19-24]}$; 利用 $6 \mathrm{~S}$ 模型, 建立 7 维大气校正查找表, 确定大气 校正系数; 利用该大气校正系数, 将 GF-2 表观反射 率转换为地表反射率。

\section{2 结果与讨论}

\section{1 大气校正对地物光谱对比分析}

本试验区选择敦煌辐射定标场, 位于扇形冲积面 上, 地域开阔, 地表平坦且均匀, 植被覆盖少, 可认 为是均匀朗伯表面, 如图 4 所示。选取 2015 年 8 月 23 日敦煌地区一景 GF-2 影像和同步过境的 MODIS 影像进行大气校正, 并将反演的表观反射率、地面反 射率与地面实测数据进行对比。

卫星观测信号的响应依赖于卫星波段的光谱响 应函数, 而地面观测的高光谱反射率数据是连续的 (如图 5(a))。为此, 将光谱仪测量获得的高光谱反射 率数据进行积分, 转换为卫星波段的等效反射率 (如 图 5(b))。转换模型如下:

$$
R_{\mathrm{eq}}=\frac{\int R(\lambda) f(\lambda) \mathrm{d} \lambda}{\int f(\lambda) \mathrm{d} \lambda}
$$

式中: $R_{\mathrm{eq}}$ 为卫星波段等效测量反射率; $R(\lambda)$ 为光谱仪 测量获得的高光谱反射率; $f(\lambda)$ 为卫星波段光谱响应 函数。

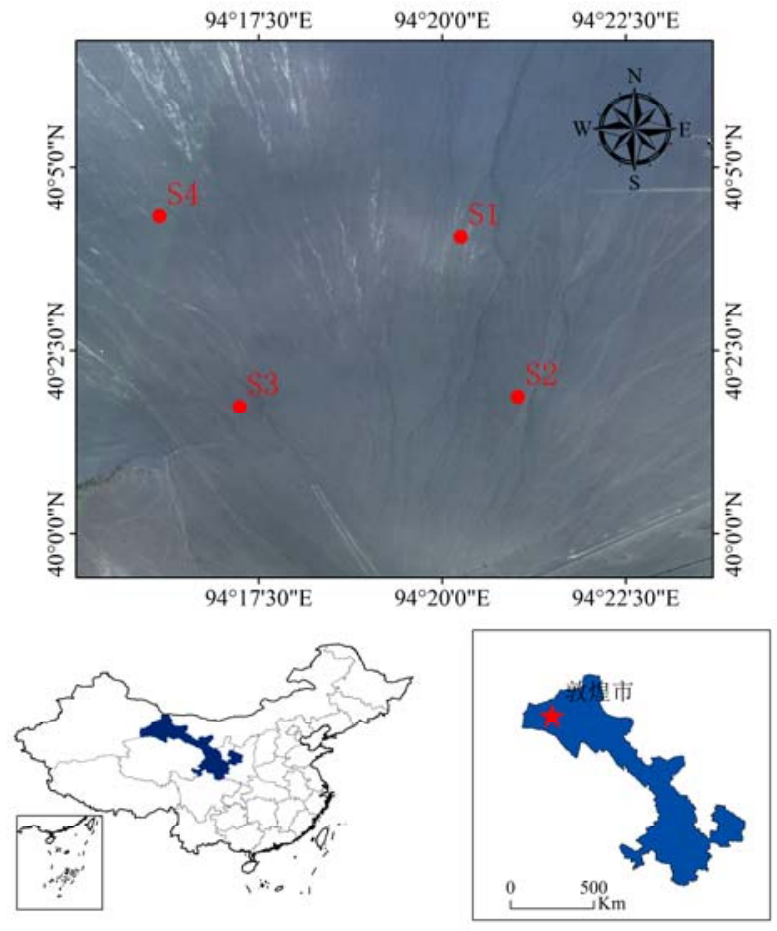

图 4 研究区地面观测点位

Fig.4 Ground observation sites in the study area

将 4 个实测点位置对于影像的表观辐亮度值使用 上述模型反演得到的表观反射率、地表反射率和实测 数据进行对比, 对比结果如图 6 所示。

如图所示, 从对比结果可以发现, GF-2 表观反射 率数据, 经过大气校正之后得到其地表反射率，蓝波 段降低, 红和红外波段明显提高, 经过大气校正后, 影像上地物反射率与地面实测反射率的吻合程度较 高, 可以更真实地反映地物反射特征。

表 4 列出了 4 个验证点的实测地表反射率和大气 校正前后反射率的对比，并且对大气校正前后反射率 与实测地表反射率求取相对误差。从表中不难发现, 校正前最大相对误差为 S3 蓝波段, 高达 $42.4 \%$, 经 过大气校正，相对误差减小至 $13.2 \%$, 且最小相对误 差为 $\mathrm{S} 1$ 的绿波段，仅为 $0.9 \%$ 。与大气校正前反射率 相对误差对比，大气校正之后反射率与实测数据之间 的差距明显缩小，大气校正效果明显。

\section{2 大气校正对 NDVI 的影响}

在遥感应用领域，植被指数已广泛用来定性和定 量评价植被覆盖及其生长活力。植被指数有明显的地 域性和时效性，受植被本身、环境、大气等条件的影 响, NDVI 可以用来检验大气校正的效果。为了验证 GF-2 数据大气校正的效果, 对校正前后的归一化植被 指数 NDVI 进行比较。NDVI 计算公式如下: 


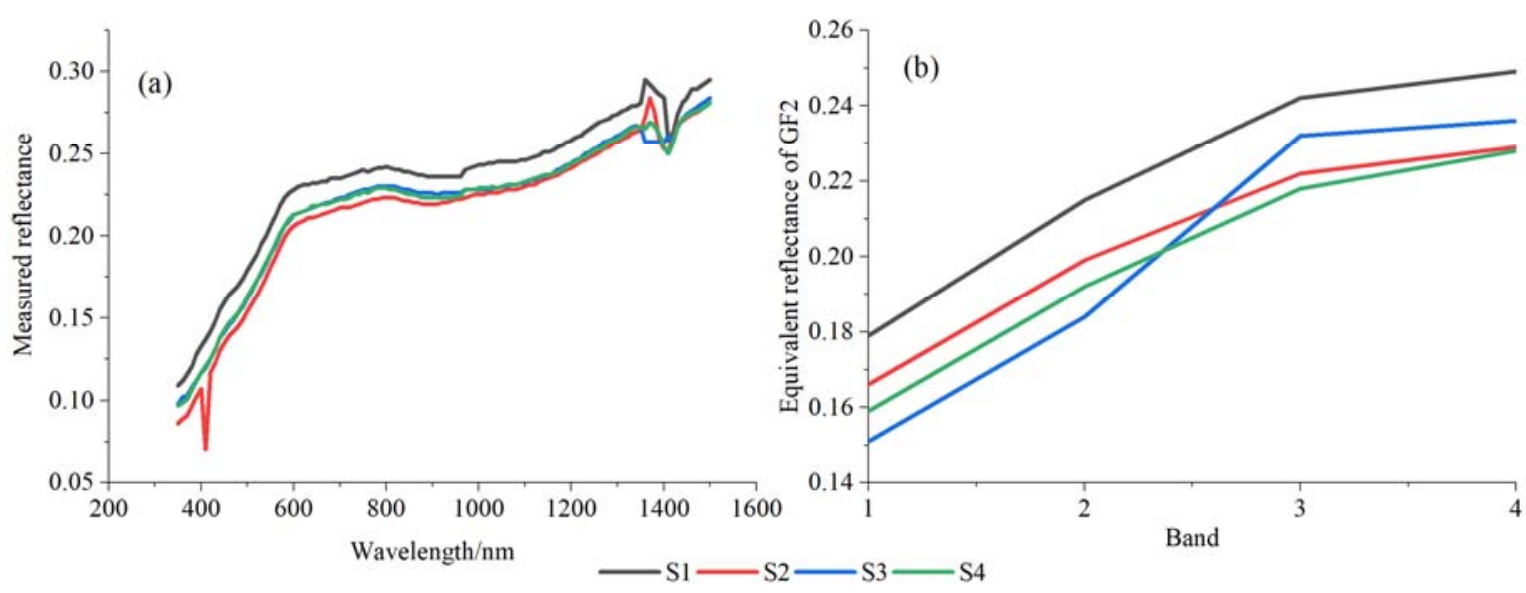

(a) 实测反射率 (a) Measured reflectance (b) GF2 传感器波段等效后的反射率 (b) Equivalent reflectance of GF2 sensor 图 5 实测光谱数据与卫星波段等效后实测反射率

Fig.5 In-situ measured spectra data and equivalent reflectance data of satellite
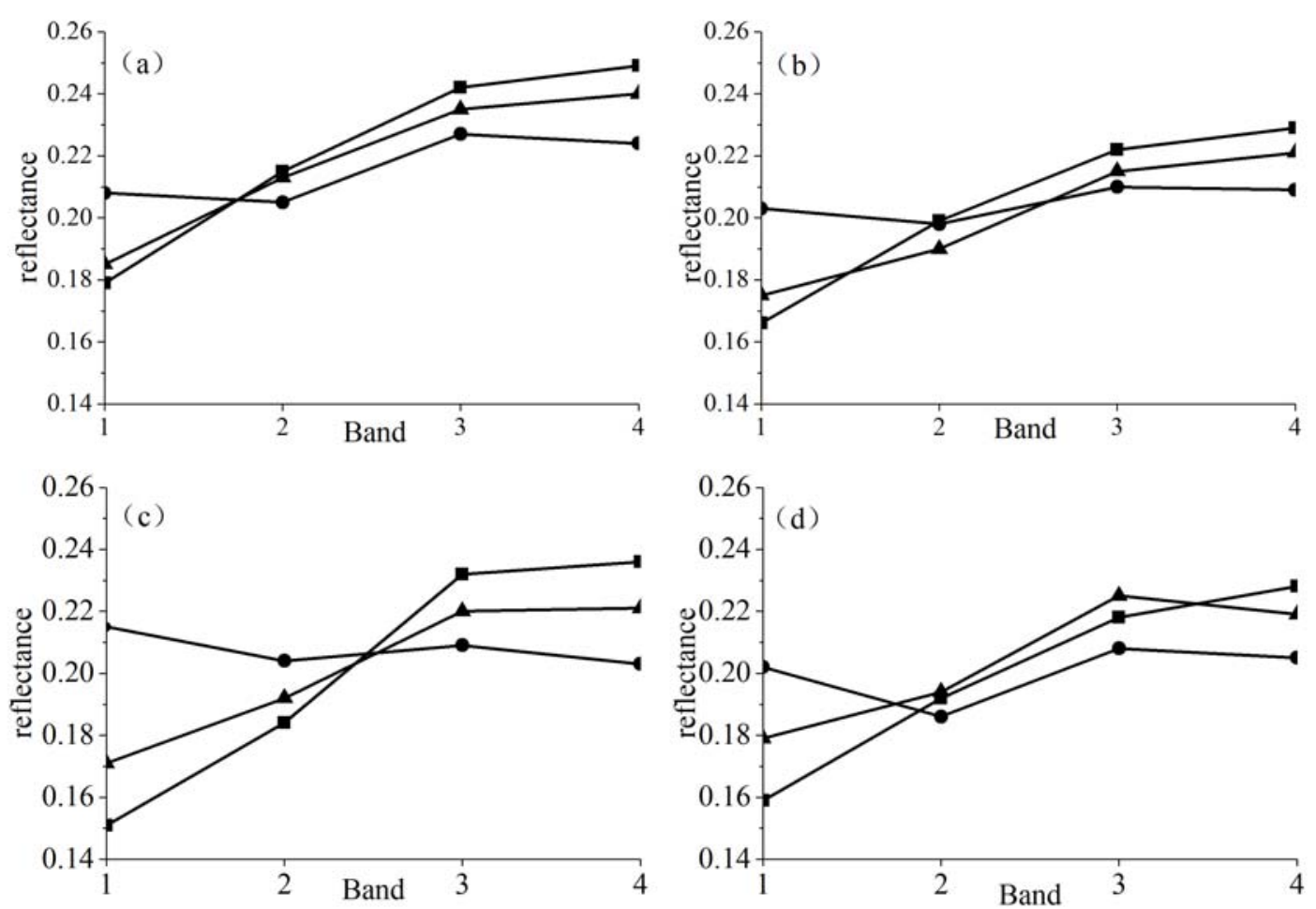

$\longrightarrow$ Measured data $\longrightarrow$ Before atmospheric correction $\longrightarrow$ After atmospheric correction

图 6 高分二号影像大气校正前后反射率比较: 图(a) (d)分别代表 $\mathrm{S} 1 \sim \mathrm{S} 4$ 四个点反射率

Fig.6 Comparison of the TOA (top of atmosphere) reflectance of GF-2 image before and after the atmospheric correction:

(a)-(d) represent the reflectance of the sample from S1 to S4

$$
\mathrm{NDVI}=\frac{\rho_{\text {nir }}-\rho_{\text {red }}}{\rho_{\text {nir }}+\rho_{\text {red }}}
$$

式中: $\rho_{\text {nir }}$ 和 $\rho_{\text {red }}$ 分别表示近红外和红波段的反射率。

选取遥感图像中的植被、水体和戈壁 3 种地物 的反射率, 根据上述公式, 计算得到 3 种地物的
NDVI, 如图 7 所示。3 种地物的 NDVI 在大气校正 前后发生了变化, 尤其是植被的 NDVI 增幅最大, 可以看出大气校正增大了 NDVI 提取植被信息的能 力, 经过大气校正处理, 可以有利于区分植被与其 他地物之间的差异。 


\section{3 结论与展望}

本文利用 $6 \mathrm{~S}$ 大气辐射传输模型建立的 7 维查找 表, 结合 MODIS 表观反射率数据, 使用改进后的暗 像元法反演气溶胶光学厚度; 依据辐射传输模型, 建 立大气校正系数查找表, 实现对 GF-2 影像数据快速 有效地大气校正。得到如下结论:

\section{表 4 高分二号影像大气校正前后反射率比较}

Table 4 Comparison the TOA reflectance of GF-2 image before and after the atmospheric correction

\begin{tabular}{|c|c|c|c|c|c|c|c|c|c|c|c|}
\hline Sample & item $^{a}$ & Blue & Green & Red & $\begin{array}{c}\text { Near } \\
\text { infrared }\end{array}$ & Sample & item $^{\mathrm{a}}$ & Blue & Green & Red & $\begin{array}{c}\text { Near } \\
\text { infrared }\end{array}$ \\
\hline \multirow{5}{*}{$\mathrm{S} 1$} & MR & 0.179 & 0.215 & 0.242 & 0.249 & \multirow{5}{*}{$\mathrm{S} 3$} & MR & 0.151 & 0.184 & 0.232 & 0.236 \\
\hline & BAC & 0.208 & 0.205 & 0.227 & 0.224 & & BAC & 0.215 & 0.204 & 0.209 & 0.203 \\
\hline & $\mathrm{AAC}$ & 0.185 & 0.213 & 0.235 & 0.240 & & $\mathrm{AAC}$ & 0.171 & 0.192 & 0.220 & 0.221 \\
\hline & REBAC $/ \%$ & 16.2 & 4.7 & 6.2 & 10.0 & & REBAC $/ \%$ & 42.4 & 10.9 & 9.9 & 14.0 \\
\hline & REAAC $/ \%$ & 3.4 & 0.9 & 2.9 & 3.6 & & REAAC $/ \%$ & 13.2 & 4.3 & 5.2 & 6.4 \\
\hline \multirow{5}{*}{$\mathrm{S} 2$} & MR & 0.166 & 0.199 & 0.222 & 0.229 & \multirow{5}{*}{ S4 } & MR & 0.159 & 0.192 & 0.218 & 0.228 \\
\hline & $\mathrm{BAC}$ & 0.203 & 0.198 & 0.210 & 0.209 & & BAC & 0.202 & 0.186 & 0.208 & 0.205 \\
\hline & $\mathrm{AAC}$ & 0.175 & 0.190 & 0.215 & 0.221 & & AAC & 0.179 & 0.194 & 0.225 & 0.219 \\
\hline & REBAC/\% & 22.3 & 0.5 & 5.4 & 8.7 & & REBAC $/ \%$ & 27.0 & 3.1 & 4.6 & 10.1 \\
\hline & REAAC $/ \%$ & 5.4 & 4.5 & 3.2 & 3.5 & & REAAC $/ \%$ & 12.6 & 1.0 & 3.2 & 3.9 \\
\hline
\end{tabular}

${ }^{\mathrm{a}} \mathrm{MR}$ : Measured reflectance; BAC: Before atmospheric correction; AAC: After atmospheric correction; REBAC: Relative error before atmospheric correction; REAAC: Relative error after atmospheric correction.

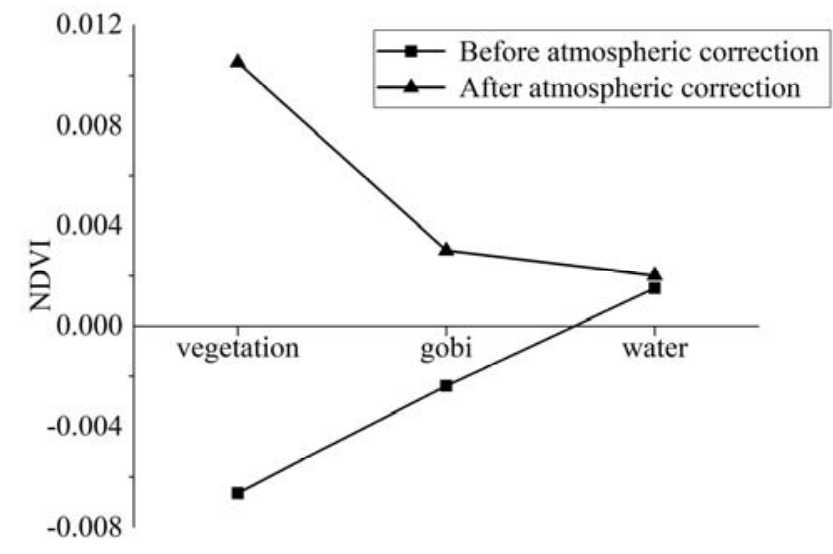

图 7 大气校正前后典型地物 NDVI

Fig.7 NDVI of typical terrain before and after atmospheric correction

GF-2 卫星经过大气校正后, 水体、戈壁和植被的 NDVI 均有变化; 大气校正后的 NDVI 大大增强了植 被信息反差，突出了 GF-2 卫星植被信息区分能力。

本研究区域地域开阔, 地表平坦且均匀, 假设 其为均匀朗伯表面, 没有考虑地物的二向反射特性。 在今后研究中, 将考虑二向反射模型（bi-direction reflectance distribution function, BRDF), 临近像元
(周围环境的反射) 和交叉辐射项对结果的影响, 提 高结果的精度, 使得该方法可以用于表面更加复杂 的区域。

\section{参考文献：}

[1] 王忠武, 刘顺喜, 戴建旺, 等. “高分二号”卫星多光谱与全色影像配准 策略[J]. 航天返回与遥感, 2015, 36(4): 48-53.

WANG Zhongwu, LIU Shunxi, DAI Jianwang, et al. Registration strategy for GF-2 satellite multispectral and panchromatic images[J]. Spacecraft Recovery \& Remote Sensing, 2015, 36(4): 48-53.

[2] 李玮, 康晓光, 陈雷. 一种 MODIS 遥感图像大气校正的快速算法 [J]. 信号处理, 2007, 23(5): 751-754.

LI Wei, KANG Xiaoguang, CHEN Lei. A fast atmospheric correction algorithm for MODIS remote sensing images[J]. Signal Processing, 2007, 23(5): 751-754.

[3] 阿布都瓦斯提, 秦其明, 朱黎江. 基于 $6 \mathrm{~S}$ 模型的可见光、近红外遥感 数据的大气校正 [J]. 北京大学学报, 2004, 40(4): 611-618.

Ghulam A, QIN Qiming, ZHU Lijiang. 6S model based atmospheric correction of visible and near-infrared data and sensitivity analysis[J]. Acta Scientiarum Naturalium Universitatis Pekinensis, 2004, 40(4): 611-618.

[4] Kaufman Y J, Remer L. Detection of forest using mid-IR reflectance: an 
application for aerosol studies[J]. IEEE Transactions on Geo science and Remote Sensing, 1994, 32(3): 672-684.

[5] 元雪勇, 田庆久. 光学遥感大气校正研究进展 [J]. 国土资源遥感, 2005, 25(4): 1-6.

QI Xueyong, TIAN Qingjiu. The advances in the study of atmospheric correction for optical remote sensing[J]. Remote Sensing for Land \& Resources, 2005, 25(4): 1-6.

[6] 刘兆亮, 麻金继. 基于 MODIS 数据反演高反射率地区气溶胶光学厚 度的方法研究 $[J]$. 大气与环境光学学报, 2012, 7(5): 358-363.

LIU Zhaoliang, MA Jinji. Retrieval of aerosol optical thickness over bright surface areas using MODIS data[J]. Journal of Atmospheric and Environmental Optics, 2012, 7(5): 358-363.

[7] Turner R E, Spencer M M. Atmospheric Model for Correction of Spacecraft Data[Z/OL]//2002: https://ui.adsabs. harvard.edu/abs/ 1972 rse..conf..895T/abstract.

[8] Gordon H R. Atmospheric correction of ocean color imagery in the earth observing system era[J]. Journal of Geophysical Research, 1997, 102(D14): 17081-17106.

[9] 赵秀娟, 陈长和, 张武, 等. 利用 MODIS 资料反演兰州地区气溶胶光 学厚度[J]. 高原气象, 2005, 24(1): 97-103.

ZHAO Xiujuan, CHEN Changhe, ZHANG Wu, et al. 2005. Retrieval of aerosol optical depth over Lanzhou area using MODIS data[J]. PLATEAU METEOROLOGY, 24(1): 97-103.

[10] 李庆利, 薛永祺, 王建宇, 等. PHI 高光谱图像的大气校正算法 [J]. 红 外与毫米波学报, 2006, 25(4): 316-320.

LI Qingli, XUE Yongqi, WANG Jianyu, et al. Atmospheric correction of PHI hyper spectral imagery[J]. Journal of Infrared and Millimeter Waves, 2006, 25(4): 316-320.

[11] 彭光雄, 何宇华, 李 京, 等. 中巴地球资源 02 星 $\mathrm{CCD}$ 图像交叉定 标与大气校正研究[J]. 红外与毫米波学报, 2007, 26(1):22-26.

PENG Guangxiong, HE Yuhua, LI Jing, et al. Study on CBERS-2's CCD image cross calibration and atmospheric correction[J]. Journal of Infrared and Millimeter Waves, 2007, 26(1): 22-26.

[12] 张璐, 施润和, 徐永明, 等. 国产遥感传感器大气层外波段平均太阳 光谱辐照度计算 $[J]$. 地球信息科学, 2014, 16(4): 624-627.

ZHANG Lu, SHI Runhe, XU Yongming, et al. Calculation of mean solar exoatmospheric irradiance of several sensors onboard of Chinese domestic remote sensing satellites[J]. Geo-Information Science, 2014, 16(4): 624-627.

[13] Kaufman Yoram J, Andrew E Wald, Lorraine A Remer, et al. The MODIS $2.1 \mu \mathrm{m}$ channel-correlation with visible reflectance for use in remote sensing of aerosol[J]. IEEE Trans Geos Remote Sensing, 1997, 35(5):1286-1298.

[14] Robert H, Wayne L, Don L, et al. Cosmic ray and hot pixel removal from STIS CCD images[J]. The HST Calibration Workshop with a New Generation of Instruments, 1997: 120-126.
[15] Anders Ångström. The parameters of atmospheric turbidity[J]. Tellus, 1964, 16(1): 64-76.

[16] 郭广猛. 关于 MODIS 卫星数据的几何校正方法 $[\mathrm{J}]$. 遥感信息, 2002(3): 26-28.

GUO Guangmeng. Geometric calibration of MODIS[J]. Remote Sensing Information, 2002(3): 26-28.

[17] 张怡, 何政伟, 薛东剑. MODIS 数据预处理方法[J]. 地理空间信息, 2013, 11(3): 49-50.

ZHANG Yi, HE Zhengwei, XUE Dongjian. The pretreatment method of MODIS data[J]. Geospatial Information, 2013, 11(3): 49-50.

[18] 杜启胜, 刘志平, 王新生, 等. 基于 ENVI 的 MODIS 数据预处理方法 [J]. 地理空间信息, 2009, 7(4): 98-100.

DU Qisheng, LIU Zhiping, WANG Xinsheng, et al. Method for MODIS data pre-processing based on ENVI[J]. Geospatial Information, 2009, 7(4): 98-100.

[19] 范娇, 郭宝峰, 何宏昌. 基于 MODIS 数据的杭州地区气溶胶光学厚 度反演 $[\mathrm{J}]$. 光学学报, 2015, 35(1): 1001-1009.

FAN J, GUO Baofeng, HE Hongchang. Retrieval of aerosol optical thickness with MODIS data over Hangzhou[J]. Acta Optica Sinica, 2015, 35(1): 1001-1009.

[20] 王玲, 田庆久, 李珊珊. 利用 MODIS 资料反演杭州市 $500 \mathrm{~m}$ 分辨率 气溶胶光学厚度 $[\mathrm{J}]$. 遥感信息, 2010 (3): 50-54.

WANG Ling, TIAN Qingjiu, LI Shanshan. Retrieval of $500 \mathrm{~m}$ spatial resolution aerosol optical thickness with MODIS data over Hangzhou[J]. Remote Sensing Information, 2010(3): 50-54.

[21] 任佳, 王振会, 孙林, 等. 基于 MODIS 数据反演江浙皖地区气溶胶 光学厚度 $[\mathrm{J}]$. 环境科学与技术, 2010,33(8): 167-171.

REN Jia, WANG Zhenhui, SUN Lin, et al. Retrieval of aerosol optical depth over Jiangsu-Zhejiang-Anhui areas using MODIS data[J]. Enviromental Science \& Technology, 2010, 33(8): 167-171.

[22] 赵志强, 李爱农, 边金虎, 等. 基于改进暗目标法山区 HJ CCD 影像 气溶胶光学厚度反演 $[\mathrm{J}]$. 光谱学与光谱分析, 2015, 35(6): 1479-1487. ZHAO Zhiqiang, LI Ainong, BIAN Jinhu, et al. An improved DDV method to retrieve AOT for HJ CCD image in typical mountainous areas[J]. Spectroscopy and Spectral Analysis, 2015, 35(6): 1479-1487.

[23] 李微, 郭锡杰, 刘远, 等. 基于改进黑暗像元法的环境一号 CCD 数据 海岸带区域大气校正研究 [J]. 海洋科学进展, 2015, 33(4): 484-491.

LI Wei, GUO Xijie, LIU Yuan, et al. Improved dark-objects atmospheric correction algorithm for coastal regions based on HJ-1 CCD data[J]. Advances in Marine Science, 2015, 33(4): 484-491.

[24] 郑伟, 曾志远. 遥感图像大气校正的黑暗像元法 $[\mathrm{J}]$. 国土资源遥感, 2005(1): 8-11.

ZHENG Wei, ZENG Zhiuan. Dark-object methods for atmospheric correction of remote sensing image[J]. Remote Sensing for Land \& Resources, 2005(1): 8-11. 